\title{
BMJ Open Femoral Nerve Block Intervention in Neck of Femur Fracture (FINOF): a randomised controlled trial
}

\author{
Martin Rowlands, ${ }^{1}$ Gerrie van de Walt, ${ }^{2}$ Jim Bradley, ${ }^{3}$ Alexa Mannings, ${ }^{1}$ \\ Sarah Armstrong, ${ }^{4}$ Nigel Bedforth, ${ }^{3}$ Iain K Moppett, ${ }^{5}$ Opinder Sahota ${ }^{6}$
}

To cite: Rowlands M, Walt Gv, Bradley J, et al. Femoral Nerve Block Intervention in Neck of Femur Fracture (FINOF): a randomised controlled trial. BMJ Open 2018;8:e019650. doi:10.1136/ bmjopen-2017-019650

- Prepublication history for this paper is available online. To view these files, please visit the journal online (http://dx.doi. org/10.1136/bmjopen-2017019650).

Received 19 September 2017 Revised 5 January 2018 Accepted 2 February 2018

Check for updates

${ }^{1}$ Department of Anaesthesia, Sheffield Teaching Hospitals, Sheffield, UK

${ }^{2}$ Department of Anaesthesia, Royal Derby Hospital, Derby, UK ${ }^{3}$ Department of Anaesthesia, Queen's Medical Centre

Nottingham University Hospitals, Nottingham, UK

${ }^{4}$ School of Medicine, Faculty of Medicine \& Health Sciences, University of Nottingham, Nottingham, UK

${ }^{5}$ Division of Clinical Neuroscience, Anaesthesia and Critical Care Group, University of Nottingham, Queen's Medical Centre, Nottingham University Hospitals, Nottingham, UK

${ }^{6}$ Department of Healthcare of Older People, Nottingham University Hospitals NHS Trust, Nottingham, UK

Correspondence to Professor Opinder Sahota; opinder.sahota@nuh.nhs.uk

\section{ABSTRACT}

Objective Fractured neck of femur is a severely painful condition with significant mortality and morbidity. We investigated whether early and continuous use of femoral nerve block can improve pain on movement and mobility after surgery in older participants with fragility neck of femur fracture.

Design Prospective single-centre, randomised controlled pragmatic trial.

Setting Secondary care, acute National Health Service Trust, UK.

Participants Participants admitted with a history and examination suggesting fractured neck of femur.

Intervention Immediate continuous femoral nerve block via catheter or standard analgesia.

Outcome measures Primary outcome measures were Cumulative Dynamic Pain score and Cumulated Ambulation Score from surgery until day 3 postoperatively. Secondary outcome measures included pain scores at rest, cumulative side effects (nausea and constipation), quality of life (measured by EuroQOL $5 \mathrm{D}$ instrument (EQ5D) score) at day 3 and day 30 , and rehabilitation outcome (measured by mobility score).

Results 141 participants were recruited, with 23 excluded. No significant difference was detected between Cumulative Dynamic Pain Score (standard care $(n=56)$ vs intervention $(n=55) 20$ (IQR 15-24) vs 20 (15-23), $\mathrm{p}=0.51$ ) or Cumulated Ambulation Score (standard care vs intervention $6(5-9)$ vs $7(5-10), p=0.76)$. There were no statistically different differences in secondary outcomes except cumulative pain at rest: $5(0.5-6.5)$ in the standard care group and $2(0-5)$ in the intervention group ( $p=0.043$ ).

Conclusions Early application of continuous femoral nerve block compared with standard systemic analgesia did not result in improved dynamic pain score or superior postoperative ambulation. This technique may provide superior pain relief at rest. Continuous femoral nerve block did not delay initial control of pain or mobilisation after surgery.

Trial registration number ISRCTN92946117; Pre-results.

\section{INTRODUCTION}

There are approximately 75000 fragility hip fractures in the UK each year, with adverse effects on life expectancy, ${ }^{1-3}$ quality of life $^{45}$ and significant health and social care

\section{Strengths and limitations of this study}

- Study designed to evaluate the role of continuous femoral nerve blockade instituted preoperatively and maintained up to 48 hours postoperatively to improve pain on movement and mobility after surgery.

- Relatively lower infusion rate may not have provided sufficient spread of local anaesthetic, and a different dose regimen may have given a different result

- We did not include patients with cognitive impairment who may have more to benefit from regional anaesthetic techniques.

costs. ${ }^{67}$ In the UK, postoperative mortality ranges between $7 \%$ and $11 \%$ at 1 month, $16 \%$ and $28 \%$ at 6 months and $22 \%$ and $37 \%$ at 1 year. ${ }^{18}$ In-hospital postoperative morbidities are a more frequent occurrence $(17 \%-50 \%)$ and often complex. A significant proportion of hip fracture survivors has decreased ability to perform activities of daily living; $50 \%$ do not regain their prefracture functional status and $10 \%-20 \%$ of those admitted from home require long-term institutional care. ${ }^{8}$ Accurate identification, quantification and care of short-term morbidity after hip fracture surgery are important since these conditions: are strongly associated with mortality and length of hospital stay ${ }^{9}$; impact long-term survival $^{10}$ and resource management.

These fractures result in severe pain and optimising pain control is both important and problematic in this older group of patients. Standard care systemic analgesia paracetamol (acetaminophen) is a good first-line treatment ${ }^{11} 12$ but is insufficient to control severe pain. Non-steroidal anti-inflammatory drugs are not recommended in this patient population. ${ }^{13}$ Opioids are widely used but may be ineffective for pain on movement. Furthermore, their use is associated with potentially serious side effects such as confusion, nausea and constipation-all of which may 
significantly impact on rehabilitation following fractured neck of femur. ${ }^{1415}$

Regional anaesthetic techniques have the theoretical advantage of avoiding the systemic effects of opioid analgesia, while providing analgesia that targets pain both at rest (static) and on movement (dynamic). ${ }^{12}$ Various peripheral nerve blocks have been described for use in hip fracture and are increasingly being used in the UK. ${ }^{416}$ Of these, femoral and fascia iliaca compartment blocks are the most commonly performed. However, many of these studies have generally focused on short-term analgesic effects of single-shot nerve blocks. Pain on movement following hip fracture lasts for at least the first few days, before and after surgery, therefore single-shot nerve blocks may have limited patient benefit. While pain relief per se is clearly of value, the additional beneficial effects of pain relief both directly and indirectly through avoidance of opioids have not been well investigated. No study has examined the effects of continuous femoral nerve blockade instituted preoperatively compared with traditional (standard care) medication on pain, rehabilitation and adverse events. We, therefore, undertook a randomised, controlled trial comparing early (preoperatively) continuous use of femoral nerve block to standard oral analgesic therapy on pain and mobility after surgery in older participants with fragility neck of femur fracture.

\section{METHODS}

This was a pragmatic, two-arm randomised controlled trial in a single centre. The study was registered with EudraCt (ref: 2010-023871-25 (17/02/2011)) and ISRCTN: ISRCTN92946117. There was a delay in trial registration due to administration oversight, but no changes in the protocol were made between first patient recruitment and trial registration.

\section{Participants}

We recruited participants aged 70 years and over admitted directly to the Emergency Department (ED) of Queen's Medical Centre, Nottingham University Hospitals NHS Trust, UK (Monday-Friday, 08:00-18:00), with a suspected fractured neck of femur. Patients had to be without significant cognitive impairment (Abbreviated Mental score of $\geq 7$ out of 10$)^{17}$ and therefore able to provide informed consent, resident in their own home or warden aided flat, with a New Mobility Score of 3 or more prior to presentation to ED (indicating independent indoor ambulation). ${ }^{18}$

Exclusion criteria included prefracture hospitalisation, contraindications to femoral nerve block analgesia, regular prefracture opioid or glucocorticoid therapy, alcohol or substance abuse, documented serious adverse reaction to morphine, restrictions to their postoperative mobilisation or already participating in another clinical trial and participants, who in the opinion of the investigator, had any condition which would adversely affect the study.
Consent was undertaken as a two-stage process. Initial witnessed verbal consent was obtained by investigators in the ED. This was to allow rapid consent and application of an initial block before participants were transferred for X-ray. Formal written consent was then obtained 24 hours later. In participants who developed confusion and were therefore deemed not to have capacity to consent at 24 hours, assent was sought from the next of kin or first nominated point of contact. If capacity was regained, formal written consent was obtained from the participant at this time.

\section{Intervention}

Participants were randomised to intervention (active) or standard care. The intervention was a femoral nerve block using $0.5 \mathrm{~mL} / \mathrm{kg}$ of $0.25 \%$ levobupivacaine (Chirocaine, Abbott Pharmaceuticals, Maidenhead, UK) up to a maximum of $30 \mathrm{~mL}$. This was provided immediately after verbal consent and was performed under ultrasound guidance by an anaesthetic research fellow in ED. A $50 \mathrm{~mm}$ linear high-frequency ultrasound transducer (S-Nerve or Micromaxx, FUJIFILM Sonosite, London, UK) was placed in the groin crease and the femoral nerve identified lateral to the femoral artery and just deep to fascia iliaca. A 50 or $100 \mathrm{~mm}$ short-bevelled regional block needle (Stimuplex A, B.Braun, Melsungen, Hessen, Germany) was inserted under the transducer 'in-plane' with the ultrasound beam in a lateral to medial direction and local anaesthetic injected around the nerve as described above. Following confirmation of fracture of the femoral neck, participants had a femoral nerve catheter sited using the same ultrasound-guided approach described above, under aseptic conditions in a 'block room' during transfer of the participant from ED to the orthopaedic ward. The perineural catheter (Contiplex Tuohy, B.Braun) was positioned under direct vision and the position deep to the nerve confirmed by injection of a small bolus of normal saline. The catheters were then tunnelled subcutaneously towards the anterior superior iliac spine, away from the expected operative site and fixed to the skin using glue (Histoacryl, B-Braun Medical, Sheffield, UK) and a transparent dressing (Tegaderm 3M Healthcare, Bracknell, UK). The nerve block was then maintained with an infusion of $0.2 \%$ ropivacaine at $5 \mathrm{~mL} /$ hour (Naropin, AstraZeneca, Luton, UK) by means of an elastomeric pump (Surefuser+ 250, Nipro Europe, Breda, The Netherlands) for 48 hours after surgery. The blocks were all performed by consultant-level and senior trainee-level research fellows, all with over 5 years of regional anaesthesia experience.

Participants in the standard care group received titrated intravenous morphine to a pain score of 5 or less at rest (verbal rating 10-point scale) before transfer to X-ray. All other care protocols were identical between the standard care and intervention group. Standard care was in accordance with national guidelines. ${ }^{13} 1920$ All participants were prescribed regular paracetamol ( $1 \mathrm{~g}$ in every 6 hours) and tramadol (50-100 $\mathrm{mg}$ in every 6 hours). Breakthrough 
analgesia was oral morphine liquid (10-20 mg up to 4 hourly) and was available for both groups as required.

Surgical fixation was determined by the operative orthopaedic surgeon following multidisciplinary review, in line with national recommendations. ${ }^{1320}$ The anaesthetic technique was left to the discretion of the operating anaesthetist. Standard care participants were permitted to have a single-shot femoral nerve block, also at the discretion of the operating anaesthetist as it was felt unethical to deprive participants of this commonly used technique. ${ }^{4}$ Intervention group participants were permitted a catheter bolus of local anaesthetic. The choice of spinal or general anaesthesia was at the discretion of the attending anaesthetist.

\section{Primary outcome measures}

Our primary outcome measures were the Cumulated Ambulation Score (CAS) and Cumulative Dynamic Pain Score and from day 1 to day 3 postoperatively. Baseline was defined as the first set of study-recorded data after participant consent.

The CAS is a validated mobility scoring system consisting of three functional domains scored $0-2$ for a maximum score of 6 . The domains are: getting in and out of bed; sitting to standing and walking. Each domain is scored: $0=$ unable to perform task, $1=$ can perform with assistance, $2=$ can perform independently. ${ }^{18} 21$ Participants were assessed daily, unblinded by the ward physiotherapist. The score was calculated daily for three postoperative days, giving a maximum possible score of 18 .

Cumulative Dynamic Pain Score was assessed unblinded by the research team using a 10-point verbal rating scale $(0=$ no pain, $10=$ worst pain experienced $)$. Once on each of the 3 days, the affected limb was elevated by $15^{\circ}$ and pain assessed. If attempted elevation of the leg was intolerable then it was abandoned and a score of 10 allocated. This gave a maximum score of 30 over the 3 days. The pain score at rest was assessed immediately before the dynamic pain score. Baseline pain scores were obtained immediately before randomisation.

\section{Secondary outcome measures}

Secondary outcome measures were: pain scores in the first $180 \mathrm{~min}$ following randomisation; pain scores at rest; presence of side effects (nausea and constipation); calorific and protein intake; quality of life (measured by EuroQOL 5D score (EQ-5D) (https://euroqol.org/)) at day 3 and day 30; length of acute hospital stay and rehabilitation outcome (measured by mobility score, at the time of discharge).${ }^{18}$

For constipation, Bristol stool charts were kept for three postoperative days. Constipation was deemed to have occurred if participants had no bowel movements on any of the 3 days.

Nausea and vomiting were scored on a 5-point scale: $0=$ no nausea/vomiting; $1=$ mild nausea only-no treatment required; 2=nausea only, antiemetics given; $3=$ vomiting, antiemetics given and $4=$ nausea/vomiting unresponsive to administered antiemetics. Participants who required treatment for nausea or vomiting (score of 2 or greater) were deemed to have had an episode of nausea or vomiting. Participants were considered to have experienced nausea/vomiting if they had any episodes of nausea/vomiting across any of the 3-day study period.

\section{Effectiveness of the blocks}

Effectiveness of the block was checked by assessing loss of cold sensation to ethyl chloride spray over the patella. Blocks were considered effective if cold sensation was altered or lost. Participants in the intervention group who had a catheter failure or dislodgement within the first 24 hours had their catheters resited. Dislodgements after 24 hours were switched to oral analgesia but remained in the intervention group for analysis on an intention-totreat basis.

\section{Statistical methods}

Sample size was estimated from previously published data for the mean dynamic pain scores ${ }^{22}$ and mean CAS. ${ }^{23}$ For the Cumulated Dynamic Pain Score, a sample size of 67 participants per group was estimated to detect a clinically relevant 2.5-point difference in mean scores between the two groups on a 10-point pain scale. For the CAS, sample size of 37 participants per group was estimated to be required to detect a clinically relevant 2-point difference in the mean CAS. Therefore, a total sample size of 150 (75 per arm) was estimated to allow a 2.5 difference in pain score and 1.5 difference in CAS, allowing for $10 \%$ attrition rate and assuming a two-sided significance level of $0.05 \%$ and $80 \%$ power.

Participants were randomised to intervention (active) or standard care group using a web-based randomisation service provided by the Nottingham Clinical Trials Unit. The randomisation sequence was generated using the ralloc module in Stata V.10.0 (StataCorp) statistical software; study participants were the unit of randomisation and were randomised with a 1:1 allocation using random block sizes of 2, 4 and 6 since placebo 'block catheters' were considered unethical, participants, staff and assessors were not blinded to group allocation.

Statistical analysis was undertaken on an intentionto-treat basis and conformed to a prespecified statistical analysis plan. Group allocation was blinded to the statistical team until analysis was complete. Comparison of CAS and cumulative pain scores was performed using Mann-Whitney U test. Secondary outcome variables were compared using the independent samples t-test for continuous outcomes or the Mann-Whitney $\mathrm{U}$ test if assumptions for using the t-test were not satisfied after appropriate transformations of the data had been applied. Binary and categorical data were analysed using the $\chi^{2}$ test. Data on pain on movement at 30,60 and $180 \mathrm{~min}$, and similarly pain at rest were recorded for descriptive purposes and formal statistical comparisons between groups were not performed. A significance level 
of 0.05 was used for all analyses. Fuller details of the trial protocol have been published previously. ${ }^{24}$

\section{RESULTS}

The first participant was randomised on 6 January 2012 and the final participant on 1 December 2014. Recruitment to the study was slower than anticipated for several reasons: lack of out-of-hours and weekend cover; the inclusion requirement for both mental capacity and mobility (approximately one in three patients lacked capacity on initial assessment) and the requirement for a high degree of clinical certainty of fracture. A total of 1224 patients presenting to the ED with hip pain were assessed for eligibility, of whom 1081 were excluded (225 did not fit inclusion criteria; 16 declined to participate; 842 were deemed not to have a fracture clinically). Of the remaining, 141 participants were randomised into the study. Seventy-one patients were allocated to the intervention, 12 further excluded because no hip fracture was shown on X-ray and 59 continued to the intervention. Fifty-five of these were included in the final analysis (four excluded: one death and three discontinued the intervention-1 exclusion criteria discovered at day 1 , one protocol violation, one randomised but no anaesthetic cover for block). Seventy patients were allocated to standard care, of which 11 were further excluded because no hip fracture seen on the X-ray and 59 continued within the study. Of these, 56 were included in the final analysis (three excluded due to one exclusion criteria discovered at day 1 , one protocol violation and one withdrew consent; figure 1).

The groups were well balanced at baseline (table 1). One hundred and eleven participants completed the full follow-up and were included in the primary analysis (table 2). The median Cumulative Dynamic Pain Scores

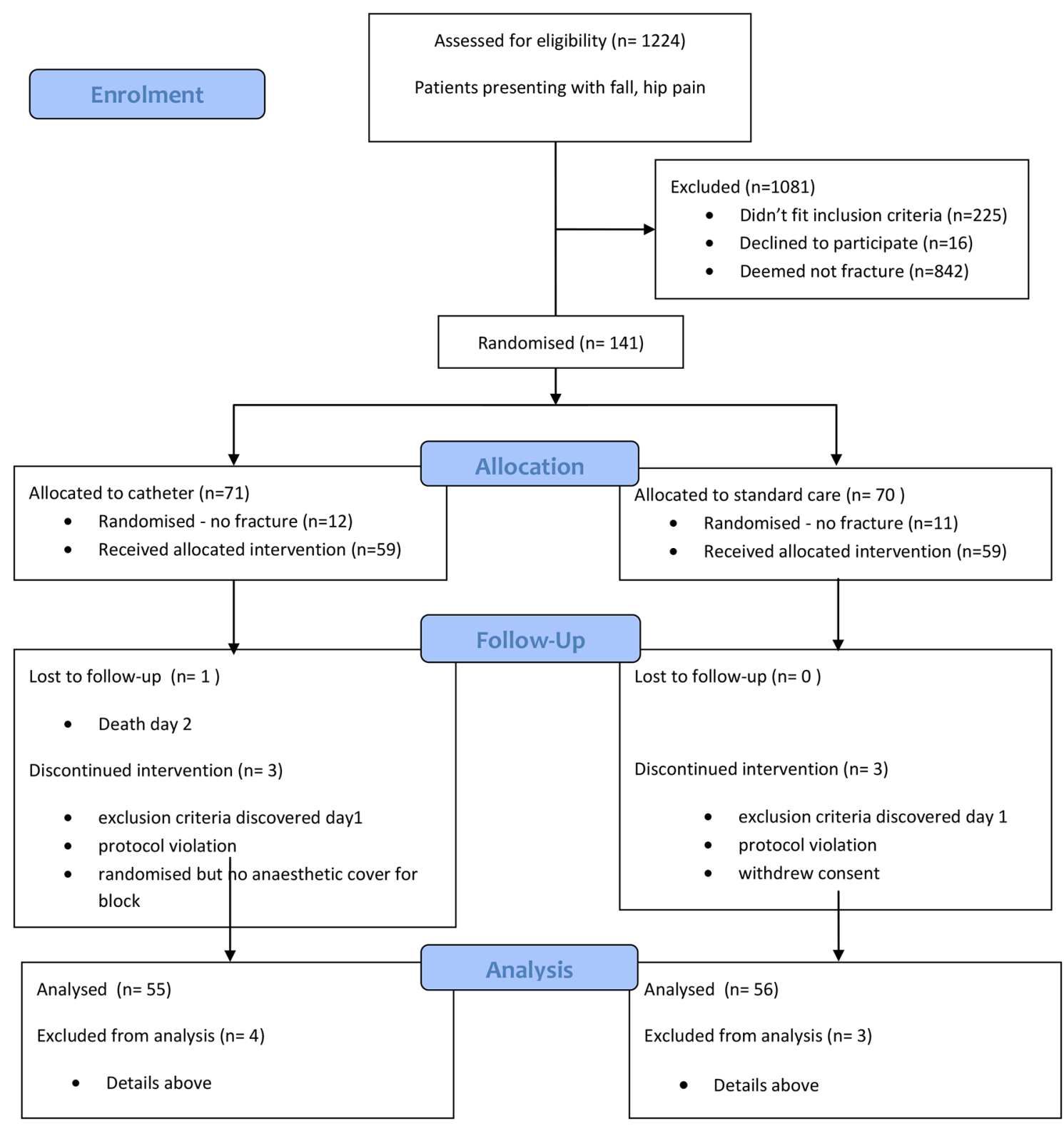

Figure 1 Consolidated Standards of Reporting Trials flow diagram. 
Table 1 Summary of patient characteristics

\begin{tabular}{|c|c|c|c|}
\hline \multicolumn{2}{|c|}{ Intervention } & $\begin{array}{l}\text { Standard } \\
\text { care }(n=59)\end{array}$ & $\begin{array}{l}\text { Intervention } \\
(n=59)\end{array}$ \\
\hline \multicolumn{2}{|c|}{ Age at inclusion } & $83.9(6.24)$ & $83.0(5.81)$ \\
\hline $84(79-90)$ & $83(79-88)$ & & \\
\hline \multirow[t]{2}{*}{ Gender } & Female & $48(76 \%)$ & $54(81 \%)$ \\
\hline & Male & $15(24 \%)$ & $13(19 \%)$ \\
\hline \multicolumn{2}{|c|}{ Body mass index (kg) } & $\begin{array}{l}23.9(3.98) \\
24(22-26)\end{array}$ & $\begin{array}{l}23.6(4.94) \\
24(20-27)\end{array}$ \\
\hline \multirow{2}{*}{$\begin{array}{l}\text { Residential } \\
\text { status }\end{array}$} & Lives alone & $32(52.5 \%)$ & $39(58 \%)$ \\
\hline & $\begin{array}{l}\text { Lives with } \\
\text { others }\end{array}$ & $29(47.5 \%)$ & $28(42 \%)$ \\
\hline \multirow[t]{5}{*}{ ASA grade } & 1 & $3(6 \%)$ & $2(4 \%)$ \\
\hline & II & $45(83 \%)$ & $40(71 \%)$ \\
\hline & III & $6(11 \%)$ & $12(21 \%)$ \\
\hline & IV & 0 & $2(4 \%)$ \\
\hline & V & 0 & 0 \\
\hline \multirow{2}{*}{\multicolumn{2}{|c|}{ Pain at rest }} & $4.92(3.18)$ & $4.28(3.14)$ \\
\hline & & $5(2-7)$ & $5(1-7)$ \\
\hline \multirow{2}{*}{\multicolumn{2}{|c|}{ Pain on movement }} & $9.59(0.99)$ & $9.65(1.14)$ \\
\hline & & $10(10-10)$ & $10(10-10)$ \\
\hline \multirow{2}{*}{\multicolumn{2}{|c|}{$\begin{array}{l}\text { New Mobility Score on } \\
\text { admission (CAS) }\end{array}$}} & $7.26(1.56)$ & $7.14(1.52)$ \\
\hline & & $7(6-9)$ & $7(6-9)$ \\
\hline \multirow{2}{*}{\multicolumn{2}{|c|}{ EuroQol EQ-5D }} & $6.52(1.53)$ & $6.57(1.79)$ \\
\hline & & $6(5-8)$ & $6(5-7)$ \\
\hline
\end{tabular}

Values are mean (SD), median (IQR or proportion).

ASA, American Society of Anaesthesiologists; CAS, Cumulated Ambulation Score.

were $20(15-24)$ in the standard care group and 19.5 (15-3) in the intervention group $(\mathrm{p}=0.51)$. The median CAS was 6 (5-9) in the standard care group and 7 (5-10) in the intervention group $(\mathrm{p}=0.76)$. Cumulative pain scores at rest were statistically different: $5(0.5-6.5)$ in the standard care group and $2(0-5)$ in the intervention group $(\mathrm{p}=0.043)$.

There were more spinal anaesthetics in the intervention group than the standard care group and this difference achieved statistical significance (general anaesthesia $29 / 56$ in the standard care group vs $39 / 55$ in the intervention group, $\mathrm{p}=0.039$ ). There were no

\begin{tabular}{llll}
\hline Table 2 & Primary outcome measures & \\
\hline Variable & $\begin{array}{l}\text { Standard care } \\
(\mathbf{n}=56)\end{array}$ & $\begin{array}{l}\text { Intervention } \\
(\mathbf{n}=55)\end{array}$ & $\begin{array}{l}\mathbf{P} \\
\text { values }\end{array}$ \\
\hline Cumulated & $7.41(3.70)$ & $7.51(3.25)$ & 0.76 \\
Ambulation Score & $6(5-9)$ & $7(5-10)$ & \\
Cumulative & $19.4(6.29)$ & $18.67(5.97)$ & 0.51 \\
Dynamic Pain & $20(15-23.8)$ & $19.5(14.5-23)$ & \\
Score & & & \\
\hline
\end{tabular}

Values are mean (SD), median (IQR or proportion). Outcomes were compared using Mann-Whitney $U$ test.
Table 3 Secondary outcome measures

\begin{tabular}{|c|c|c|c|}
\hline $\begin{array}{l}\text { Secondary } \\
\text { outcomes }\end{array}$ & $\begin{array}{l}\text { Standard care } \\
(n=56)\end{array}$ & $\begin{array}{l}\text { Intervention } \\
(\mathrm{n}=55)\end{array}$ & $\begin{array}{l}P \\
\text { values }\end{array}$ \\
\hline \multirow[t]{2}{*}{ Length of stay } & 15.9 (10.67) & $16.2(9.56)$ & \\
\hline & $14(9.25-19.5)$ & $13(10-18)$ & 0.89 \\
\hline \multirow{2}{*}{$\begin{array}{l}\text { Pain on movement } \\
\text { at } 30 \mathrm{~min}\end{array}$} & $9.62(1.29)$ & $9.11(1.45)$ & \\
\hline & $10(10-10)$ & $10(8-10)$ & \\
\hline \multirow{2}{*}{$\begin{array}{l}\text { Pain on movement } \\
\text { at } 60 \mathrm{~min}\end{array}$} & $9.4(1.59)$ & 8.84 (1.99) & \\
\hline & $10(10-10)$ & $10(8.25-10)$ & \\
\hline \multirow{2}{*}{$\begin{array}{l}\text { Pain on movement } \\
\text { at } 180 \mathrm{~min}\end{array}$} & $9.35(1.17)$ & $8.68(1.91)$ & \\
\hline & $10(9-10)$ & $9(8-10)$ & \\
\hline \multirow{2}{*}{$\begin{array}{l}\text { Pain on rest at } \\
30 \mathrm{~min}\end{array}$} & 3.64 (2.73) & $2.92(2.77)$ & \\
\hline & $3(1-5)$ & $2(0-4.5)$ & \\
\hline \multirow{2}{*}{$\begin{array}{l}\text { Pain on rest at } \\
60 \mathrm{~min}\end{array}$} & 3.28 (2.69) & $2.27(2.46)$ & \\
\hline & $3(1-5)$ & $2(0-4)$ & \\
\hline \multirow{2}{*}{$\begin{array}{l}\text { Pain on rest at } \\
180 \mathrm{~min}\end{array}$} & $2.76(2.61)$ & $2.16(2.35)$ & \\
\hline & $2(1-4)$ & $1(0-3.25)$ & \\
\hline \multirow{2}{*}{$\begin{array}{l}\text { Cumulative pain } \\
\text { score at rest }\end{array}$} & $4.92(4.75)$ & 3.16 (3.54) & \\
\hline & $5(0.5-6.5)$ & $2(0-5)$ & 0.043 \\
\hline $\begin{array}{l}\text { Presence of } \\
\text { delirium }\end{array}$ & $4 / 54$ (7.4\%) & $0 / 54(0 \%)$ & $0.118^{*}$ \\
\hline $\begin{array}{l}\text { Presence of } \\
\text { constipation }\end{array}$ & $30 / 54$ (56\%) & $25 / 50$ (50\%) & $0.57^{\star}$ \\
\hline $\begin{array}{l}\text { Presence of } \\
\text { nausea/vomiting }\end{array}$ & $6 / 56(10.7 \%)$ & $5 / 51(9.8 \%)$ & $0.877^{*}$ \\
\hline \multirow{2}{*}{$\begin{array}{l}\text { New mobility } \\
\text { score at } 30 \text { days }\end{array}$} & $2.5(2.08)$ & 2.34 (2.39) & \\
\hline & $2(0-4)$ & $2(0-3)$ & 0.49 \\
\hline \multirow{2}{*}{$\begin{array}{l}\text { EuroQol EQ-5D at } \\
30 \text { days }\end{array}$} & $8.79(2.20)$ & $8.67(2.10)$ & $0.78 \dagger$ \\
\hline & $9(7-10)$ & $8(7-10)$ & \\
\hline \multirow{2}{*}{$\begin{array}{l}\text { EuroQol EQ-5D at } \\
3 \text { days }\end{array}$} & $9.92(1.52)$ & $9.65(1.42)$ & 0.37 \\
\hline & $10(9-11)$ & $10(9-10)$ & \\
\hline
\end{tabular}

Values are mean (SD), median (IQR or proportion). The $p$ values reported were obtained from the Mann-Whitney $U$ test unless otherwise stated. Binary and categorical data were analysed using the $\chi^{2}$ test.

${ }^{*} \mathrm{P}$ values obtained from the $\chi^{2}$ test.

†P values obtained from the two-sample t-test.

significant differences in the remaining secondary outcomes (table 3). Despite the efforts of the research team, routine protein calorific intake was recorded in less than $10 \%$ of participants and therefore not reliable.

Time from randomisation to theatre was not statistically different between the two groups: standard care 22.5 
(19.6-25.2) hours, intervention 23.6 (20.1-25.6) hours, $(\mathrm{p}=0.412)$. Two participants required catheter resites and four catheters were withdrawn early due to dislodgement (after 24 hours but before 48 hours). Unfortunately, block assessment was incorrectly coded by a member of the research team for an unknown number of participants (absence of sensation was recorded as an absence of block and vice versa). Daily surveillance for block-related complications did not result in any cases of suspected nerve damage, haematoma or infection. There were no falls in the block group during the study period. One patient fell in the standard care group.

\section{DISCUSSION}

Early (preoperatively) and continuous (up to 48 hours postoperatively) femoral nerve block was not associated with improvements in Cumulative Dynamic Pain Scores or mobility from surgery to 3 days postoperatively. There was a significant difference in pain scores at rest but no observed benefit on opioid-related side effects (nausea, constipation) nor a range of surrogate markers of recovery. We have demonstrated that pain on movement is severe both before and after surgery and in the early postoperative period.

Our results seemingly run counter to current recommendations and to an extent clinical practice. It is, therefore, appropriate to critique both the intervention and the outcome measures used in the study.

\section{Pain mechanism}

Pain following fractured neck of femur is usually severe and while early assessment and treatment of pain is recommended, ${ }^{13}$ National Institute for Health and Care Excellence (NICE) guidelines only recommend nerve blocks when regular oral analgesics (paracetamol and opioids) are failing to control pain. It is also recognised by NICE that use of nerve blocks should not prevent early surgical fixation.

Pain from fractured neck of femur is complex and multifactorial involving muscle spasm, fracture movement, surgical wounds and psychosocial factors. Femoral nerve block will only impact on part of this process. The hip joint itself receives innervation from several nerves. ${ }^{25}$ A common comment from participants in the intervention arm was that pain seemed to migrate from the front of the hip to the back. This is consistent with the sensory block expected from femoral nerve block and the lack of blockade of the sciatic and probably obturator nerves. Techniques which target more of the nerves supplying the hip have shown improvements in pain, ${ }^{15162326}$ but not necessarily other endpoints.

\section{Optimal regional anaesthesia}

Precise placement of nerve sheath catheters with ultrasound guidance can provide a more effective block of the lumbar plexus than those guided by nerve stimulator. Use of the ultrasound has been shown to be more effective than increasing the volume of local anaesthetic for single-shot blocks. It was anticipated that the $5 \mathrm{~m}$ /hour infusion rate would provide effective analgesia without compromising motor function and mobility. On reflection, we accept that this lower infusion rate may not have provided sufficient spread of local anaesthetic. In addition, at the outset of study design we carefully considered the balance between analgesia and motor blockade. A different dose regimen may have given a different result; intermittent bolusing rather than continuous infusion is another possibility. Other studies of femoral nerve catheter local anaesthetic infusions have shown mixed results. Szucs $e t$ al used an infusion of $0.25 \%$ bupivacaine at $4 \mathrm{~mL} /$ hour and demonstrated reduced pain scores on passive movement at all time points up to 72 hours. ${ }^{27}$ Chaudet $e t a t^{28}$ used ropivacaine $2 \mathrm{mg} / \mathrm{mL}$ (the same as this study) but at $8 \mathrm{~mL} /$ hour (compared with $5 \mathrm{~mL} /$ hour in this study). They found no difference in pain scores between local anaesthetic and placebo infusion groups. A reduction in morphine-associated side effects (predominantly nausea) was seen despite no difference in total morphine consumption. Gille et $a t^{29}$ used the same infusion regimen as this study and found no difference in pain relief between the catheter group and a systemic analgesia group over 3 days. A retrospective database study from Helsø $e t a \hat{l}^{30}$ found no difference in morphine consumption during the first 5 days of admission between those given continuous femoral nerve blockade and those without.

Although it is possible that the greater proportion of spinal anaesthetics in the block group may have affected analgesia in the very early postoperative period, we do not think that an effect would have been observed over the three postoperative days.

More complete coverage of the hip joint would be expected from epidural analgesia. Foss et $a \hat{l}^{31}$ demonstrated a significant reduction in a 5-point pain scale with such an approach, though without an overall benefit in terms of early mobilisation or hospital discharge and with an increase in nausea. In a study comparing continuous femoral nerve blockade with participant controlled intravenous morphine and continuous epidural analgesia in elective hip surgery, no differences were found in quality of analgesia although the femoral nerve block was associated with fewer side effects. ${ }^{28}$ Other studies of similar design have not reported differences in pain scores. ${ }^{26}$ Epidural analgesia is uncommon in UK practice in this group of patients, with concerns about nursing time, catheter fall out and falls secondary to bilateral motor and proprioceptive block.

The use of a relatively high dose of tramadol might have reduced any difference seen between the groups. However, at the time of study design this was considered to be a reasonable standard of analgesia and we felt it would have been unethical to deny this to participants. We acknowledge that clinical practice has changed in the intervening period and oral morphine or oxycodone is more commonly used now. Furthermore, the reported dynamic pain scores in both groups were high. Tramadol 
does not appear to be effective for preventing severe dynamic pain.

\section{Limitations of the study}

The incorrect coding of block assessment was a result of (unnoticed) confusion between absence of sensation and presence of a block. However, even had these been coded correctly, the study was intended as a pragmatic study with all blocks and catheters placed by experienced clinicians. Dynamic pain may be a better endpoint than static pain. In common with other studies, ${ }^{27}$ our results showed that static pain is relatively well controlled following hip fracture. But, it is dynamic pain that is felt to impact on rehabilitation. Analgesia sufficient to allow movement necessary for investigations is also a NICE recommendation. ${ }^{13}$ The pain scores reported by participants in this study were considerably higher than those reported by Foss $e t a l$, both in the intervention and control groups. Given that the pathology of hip fracture is the same, we can only surmise that the interpretation of a pain score is different between older people in England and Denmark. There is reasonable evidence for the use of pain scales in older people and many similar studies have used the verbal rating scale in similar populations. ${ }^{1231-33}$

We did not collect data on morphine consumption as morphine consumption is of little direct relevance: participants are concerned with quality of analgesia and adverse effects. One of the perceived benefits to the use of nerve block techniques is the opioid-sparing effect that they provide. Unlike Chaudet $e t a l,{ }^{28}$ we found no difference in common opioid-related side effects (nausea and constipation); Helsø $e t a l^{30}$ found no morphine-sparing effect. The relatively low nausea rates in this study compared with others may explain some of this difference. The rates of delirium recorded during the study were low, with no significant difference between the groups. This may, in part be due to the exclusion of patients with dementia-a strong risk factor for delirium, and those with impaired cognitive function on admission. Our participants would therefore represent a lower risk group for delirium. We acknowledge that analgesia is not the only factor that influences postoperative mobility, and other medical conditions will have an impact. The fact that there were more spinal anaesthetics than general anaesthetics may represent a soft marker of efficacy. Standard practice in our institution is to place a femoral nerve block and then sit participants forward to place a subarachnoid block for surgical anaesthesia. Participants who are obviously in too much discomfort to sit forward are often given a general anaesthetic. The proportion of spinal anaesthetics given in the intervention group may represent participants generally being more comfortable being sat forward, but there may be other reasons.

Due to the design of the study only including patients with mental capacity and good mobility, the participants were generally somewhat fitter than the overall hip fracture population. ${ }^{34}$ The frailer population may have more to gain from better analgesia in terms of enhancing mobility and avoidance of delirium; this study was not designed to assess this. However, we failed to demonstrate an analgesic benefit and there is no plausible explanation for why femoral nerve blocks would be more effective in frailer older people.

Finally, pain from hip fracture starts at the time of injury and it may be that earlier intervention is appropriate. The time from injury to assessment in ED (and therefore trial recruitment) can be prolonged and vary considerably between individuals. Although this is a potential unmeasured confounder, we believe that randomisation is likely to have balanced the groups. Trials of fascia iliaca compartment block delivered by paramedics ${ }^{35}$ are taking place, and we await the results with interest. Although the exclusion of participants who were found not to have a fractured neck of femur meant that we did not reach our recruitment target, we do not believe further recruitment would have altered our results in a meaningful way. The lack of difference between the two groups was such that a statistically significant difference would be almost impossible to achieve.

\section{CONCLUSION}

In summary, we do not believe that the widespread use of femoral nerve blocks should be abandoned on the basis of one negative study. However, previous studies looking at pain in fractured neck of femur participants receiving femoral nerve catheters perioperatively report mixed results. ${ }^{94-27}$ Our study does not provide evidence to support the widespread introduction of femoral nerve catheters for prolonged infusion. Pain following fractured neck of femur and surgery remains an unresolved problem, with a lack of good evidence to support any single technique.

Future studies need to look at the effects of extended block of the hip in terms of rehabilitation and analgesic side effects. There may be benefit in investigating higher dose or intermittent bolus regimens. Blockade of more of the nerves supplying the hip may be able to demonstrate benefit in cumulative outcomes rather than acute analgesic effects. Early and continuous femoral nerve block is not superior to standard analgesia in terms of its effect on dynamic pain relief or cumulated postoperative ambulation. Femoral nerve block does not delay initial control of pain or mobilisation after surgery.

Acknowledgements The study team would like to acknowledge and express thanks for the support and participation of the Nottingham University Hospitals NHS Trust as well as all individuals and groups who agreed to take part. In addition, further acknowledgement is needed for the participants and families who shared with the study team their personal and often protracted experiences of hospital care and community follow-up. The study team would also like to express thanks for the support and guidance of the Trial Steering Committee (TSC): Dr Nicola Morgan and Dr Pascal Brodie. Many thanks to the research team nurses Tsvetely Angelova, Alison Watson, Anna Gibbs, Lani Patterson, Sally Lee, Leigh DeVivo without whom the study could not have been completed, and Dr Mohammed Rafi and Dr Lakshmipathy Purushuthaman who also contributed.

Contributors OS, IKM, NB and SA were involved in the design, conduct, analysis and writing of the study. MR, GvdW, JB and AM were involved in the conduct, analysis and writing of the study. 
Funding This work was supported by a grant from the National Institute of Health Research, UK. (NIHR Project Number: PB-PG-0909-19119).

Competing interests None declared.

Patient consent Obtained.

Ethics approval The study was approved by Nottingham Research Ethics Committee, Reference 10/H0408/113 (28/1/2011).

Provenance and peer review Not commissioned; externally peer reviewed.

Data sharing statement № further unpublished data are available.

Open Access This is an Open Access article distributed in accordance with the Creative Commons Attribution Non Commercial (CC BY-NC 4.0) license, which permits others to distribute, remix, adapt, build upon this work non-commercially, and license their derivative works on different terms, provided the original work is properly cited and the use is non-commercial. See: http://creativecommons.org/ licenses/by-nc/4.0/

(C) Article author(s) (or their employer(s) unless otherwise stated in the text of the article) 2018. All rights reserved. No commercial use is permitted unless otherwise expressly granted.

\section{REFERENCES}

1. NHFD. Annual report. 2017 http://www.nhfd.co.uk/20/hipfractureR nsf/docs/2017Report (accessed on 30 Oct 2017).

2. Haentjens P, Magaziner J, Colón-Emeric CS, et al. Meta-analysis: excess mortality after hip fracture among older women and men. Ann Intern Med 2010:152:380-90.

3. Marufu TC, White SM, Griffiths R, et al. Prediction of 30-day mortality after hip fracture surgery by the Nottingham Hip Fracture Score and the Surgical Outcome Risk Tool. Anaesthesia 2016;71:515-21.

4. White SM, Moppett IK, Griffiths R, et al. Secondary analysis of outcomes after 11,085 hip fracture operations from the prospective UK Anaesthesia Sprint Audit of Practice (ASAP-2). Anaesthesia 2016;71:506-14.

5. Parsons N, Griffin XL, Achten J, et al. Outcome assessment after hip fracture: is EQ-5D the answer? Bone Joint Res 2014;3:69-75.

6. Peeters CM, Visser E, Van de Ree CL, et al. Quality of life after hip fracture in the elderly: a systematic literature review. Injury 2016;47:1369-82.

7. Lawrence TM, White CT, Wenn R, et al. The current hospital costs of treating hip fractures. Injury 2005;36:88-91.

8. Smith PAC, Bardsley M. Focus on hip fracture: trends in emergency admissions for fractured neck of femur, 2001 to 2011. The Health Foundation and the Nuffield Trust, 2013. http://www.nuffieldtrust.org. uk/sites/files/nuffield/publication/131010_focus-on-hip-fracture.pdf (accessed 20 Dec 2016)

9. Howes TE, Cook TM, Corrigan LJ, et al. Postoperative morbidity survey, mortality and length of stay following emergency laparotomy. Anaesthesia 2015;70:1020-7.

10. Moonesinghe SR, Harris S, Mythen MG, et al. Survival after postoperative morbidity: a longitudinal observational cohort study. $\mathrm{Br}$ $J$ Anaesth 2014;113:977-84.

11. Foss NB, Kristensen MT, Palm H, et al. Postoperative pain after hip fracture is procedure specific. Br J Anaesth 2009;102:111-6.

12. Cuvillon P, Ripart J, Debureaux S, et al. Analgesia after hip fracture repair in elderly patients: the effect of a continuous femoral nerve block: a prospective and randomised study. Annales Françaises d'Anesthésie et de Réanimation 2007;26:2-9.

13. National Institute for Health and Care Excellence. The management of hip fracture in adults. National Clinical Guideline Centre. 2011 https://www.nice.org.uk/guidance/cg124 (accessed 27 Jun 2017).

14. Trads M, Pedersen PU. Constipation and defecation pattern the first 30 days after hip fracture. Int $J$ Nurs Pract 2015;21:598-604.
15. Carpintero P, Caeiro JR, Carpintero R, et al. Complications of hip fractures: A review. World J Orthop 2014;5:402-11.

16. Guay J, Parker MJ, Griffiths R, et al. Peripheral nerve blocks for hip fractures. Cochrane Database Syst Rev 2017;5:CD001159.

17. Hodkinson HM. Evaluation of a mental test score for assessment of mental impairment in the elderly. Age Ageing 1972;1:233-8.

18. Parker MJ, Palmer CR. A new mobility score for predicting mortality after hip fracture. J Bone Joint Surg Br 1993;75:797-8.

19. Department of Health Payment by Results Team. Payment by results guidance for 2013-14. London: Department of Health, 2013. https:// www.gov.uk/government/publications/payment-by-results-pbroperational-guidance-and-tariffs. (accessed 27 Jun 2017).

20. The care of patients with fragility fracture (The blue book). London: British Orthopaedic Association, 2012. https://www.boa.ac.uk/wpcontent/uploads/2014/12/BOAST-1.pdf. (accessed 16 Oct 2017).

21. Kristensen MT, Jakobsen TL, Nielsen JW, et al. Cumulated Ambulation Score to evaluate mobility is feasible in geriatric patients and in patients with hip fracture. Dan Med $J$ 2012;59:A4464.

22. Olsen MF, Bjerre E, Hansen MD, et al. Pain relief that matters to patients: systematic review of empirical studies assessing the minimum clinically important difference in acute pain. BMC Med 2017;15:35

23. Foss NB, Kristensen BB, Bundgaard M, et al. Fascia iliaca compartment blockade for acute pain control in hip fracture patients: a randomized, placebo-controlled trial. Anesthesiology 2007;106:773-8.

24. Sahota O, Rowlands M, Bradley J, et al. Femoral nerve block Intervention in Neck of Femur fracture (FINOF): study protocol for a randomized controlled trial. Trials 2014;15:189.

25. Birnbaum K, Prescher A, Hessler S, et al. The sensory innervation of the hip joint--an anatomical study. Surg Radiol Anat 1997;19:371-5.

26. Beaudoin FL, Nagdev A, Merchant RC, et al. Ultrasound-guided femoral nerve blocks in elderly patients with hip fractures. Am J Emerg Med 2010:28:76-81.

27. Szucs S, lohom G, O'Donnell B, et al. Analgesic efficacy of continuous femoral nerve block commenced prior to operative fixation of fractured neck of femur. Perioper Med 2012;1:4-8.

28. Chaudet A, Bouhours G, Rineau E, et al. Impact of preoperative continuous femoral blockades on morphine consumption and morphine side effects in hip-fracture patients: A randomized, placebo-controlled study. Anaesth Crit Care Pain Med 2016;35:37-43

29. Gille J, Gille M, Gahr R, et al. [Acute pain management in proximal femoral fractures: femoral nerve block (catheter technique) vs. systemic pain therapy using a clinic internal organisation model]. Anaesthesist 2006;55:414-22.

30. Helsø I, Jantzen C, Lauritzen JB, et al. Opioid Usage During Admission in Hip Fracture Patients-The Effect of the Continuous Femoral Nerve Block. Geriatr Orthop Surg Rehabil 2016;7:197-201.

31. Foss NB, Kristensen MT, Kristensen BB, et al. Effect of postoperative epidural analgesia on rehabilitation and pain after hip fracture surgery: a randomized, double-blind, placebo-controlled trial. Anesthesiology 2005;102:1197-204.

32. Singelyn FJ, Ferrant T, Malisse MF, et al. Effects of intravenous patient-controlled analgesia with morphine, continuous epidural analgesia, and continuous femoral nerve sheath block on rehabilitation after unilateral total-hip arthroplasty. Reg Anesth Pain Med 2005;30:452-7.

33. Herr KA, Garand L. Assessment and measurement of pain in older adults. Clin Geriatr Med 2001;17:457-78.

34. Johansen A, Tsang C, Boulton C, et al. Understanding mortality rates after hip fracture repair using ASA physical status in the National Hip Fracture Database. Anaesthesia 2017;72:961-6.

35. Bulger JK, Brown A, Evans BA, et al. Rapid analgesia for prehospital hip disruption (RAPID): protocol for feasibility study of randomised controlled trial. Pilot Feasibility Stud 2017;3:8. 